\title{
PERFIL EPIDEMIOLÓGICO DOS INDIVIDUOS COM GRAU DOIS DE INCAPACIDADE FISICA NOS CASOS NOVOS dE haNSENIASE, DURANTE 10 ANOS, EM GUARAPUAVA-PR
}

\author{
Epidemiological profile of grade 2 physical disability \\ individuals in new cases of leprosy, during 10 years, \\ in Guarapuava-PR
}

Sabrina Aparecida Wendler ${ }^{1}$, Vanessa Cristina Novak ${ }^{2}$, Cíntia Raquel Bim ${ }^{3}$, Aline Cristina Carrasco $^{4}$, Christiane Riedi Daniel ${ }^{5}$

1. Egressa do curso de Fisioterapia da Universidade Estadual do Centro-Oeste - UNICENTRO, GuarapuavaPR, Brasil. ORCID: https://orcid.org/0000-0002-1875-3866

2. Mestre. Docente do curso de Fisioterapia da Universidade Estadual do Centro-Oeste-UNICENTRO, Guarapuava-PR, Brasil. ORCID: http://orcid.org/0000-0002-1432-8774

3. Mestre. Docente do curso de Fisioterapia da Universidade Estadual do Centro-Oeste-UNICENTRO, Guarapuava-PR, Brasil. ORCID: http://orcid.org/0000-0001-7067-2572

4. Mestre. Docente do curso de Fisioterapia da Universidade Estadual do Centro-Oeste-UNICENTRO, Guarapuava-PR, Brasil. ORCID: http://orcid.org/0000-0002 3513-4506

5. Doutora. Docente do curso de Fisioterapia da Universidade Estadual do Centro-Oeste-UNICENTRO, Guarapuava-PR, Brasil. ORCID: http://orcid.org/0000-0002 2282-1358

CONTATO: Cíntia Raquel Bim | Rua Simeão Camargo Varela de Sá no 04 | Vila Carli | CEP: 85040०80 | Guarapuava | Paraná | E-mail: cintiabim@gmail.com

COMO CITAR: Wendler SA, Novak VC, Bim CR, Carrasco AC, Daniel CR. Perfil epidemiológico dos indivíduos com grau dois de incapacidade física nos casos novos de hanseníase, durante 10 anos, em Guarapuava-PR. R. Saúde Públ. 2018 Dez;1(2):90-100

(c) Ci) Internacional. É permitida a reprodução parcial ou total desta obra, desde que citada a fonte.

RESUMO A Hanseníase é uma patologia infectocontagiosa e crônica, possui alto poder incapacitante e representa um grave problema para a saúde pública no Brasil. OBJETIVO: Traçar o perfil dos casos novos de hanseníase, classificados com o grau 2 de incapacidade física (GIF2) no momento do diagnóstico e descrever 
as principais incapacidades causadas pela doença. MÉTODOS: Estudo epidemiológico transversal, cujo levantamento de dados foi baseado nos prontuários, registros em Livros Ata nos arquivos do Ambulatório Municipal de Pneumologia e Dermatologia Sanitária de Guarapuava-PR e no Sistema de Informação de Agravos de Notificação do Ministério da Saúde. RESULTADOS: A presença do GIF2 foi mais frequente no sexo masculino, com cor de pele branca, baixa escolaridade, classificação operacional multibacilar, forma clínica virchowiana. As principais incapacidades encontradas foram lesões tróficas ou traumáticas em pés e garra em mãos. CONCLUSÃO: Há necessidade de intensificar as ações educativas para prevenção, contribuindo para o diagnóstico precoce da doença e evitando as incapacidades.

PALAVRAS-CHAVE Hanseníase. Doenças Negligenciadas. Doença Infecciosa. Controle de Doenças Transmissíveis.

ABSTRACT Leprosy was an infectious and chronic pathology, with high incapacitating power, and represents a serious problem for public health in Brazil. OBJECTIVE: To describe the profile of new cases of leprosy classified as grade 2 physical disability (G2D) at the time of diagnosis, and to describe the main disabilities caused by the disease. METHODS: Cross-sectional epidemiological study, whose data collection was based on medical records, records in books in the archives of the Municipal Ambulatory of Pneumology and Sanitary Dermatology of Guarapuava-PR, and in the Information System of Notifiable Ministry of Health Diseases. RESULTS: The presence of GIF2 is more frequent in maleswith white skin color, low level of education, multibacillary operational classification, virchowian clinical form. The main disabilities were trophic or traumatic lesions in the foot, and claw hands. CONCLUSION: It is necessary to enhance educational actions for prevention, contributing to the early diagnosis of the disease, and avoiding disabilities.

KEYWORDS: Leprosy. Neglected Diseases. Infectious disease. Control of Communicable Diseases.

\section{INTRODUÇÃO}

$\mathbf{A}$

hanseníase é uma patologia

infectocontagiosa e crônica, causada pelo

Mycobacterium leprae (M. leprae) ou bacilo de Hansen, descrito pela primeira vez em 1868, pelo médico Amaneur Hansen1. O tempo de multiplicação do bacilo é lento, podendo durar em média de 11 a 16 dias. Já o período de incubação é longo e as diferentes manifestações clínicas da doença podem aparecer de 2 a 7 anos. O M. leprae é caracterizado por alta infectividade e baixa patogenicidade, e possui afinidade por células cutâneas e células dos nervos periféricos².
De acordo com a Organização Mundial da Saúde (OMS)3 , em 2016, 143 países reportaram 214.783 casos novos de hanseníase, o que representa uma taxa de detecção de 2,9 casos por 100 mil habitantes. No Brasil, no mesmo ano, foram notificados 25.218 casos novos, perfazendo uma taxa de detecção de 12,2 casos por 100 mil habitantes. Os focos endêmicos estão localizados em países em desenvolvimento, correlacionados às condições socioeconômicas, culturais, de escolaridade, acesso a informações e essencialmente do acesso aos serviços de saúde ${ }^{4}$. O Brasil está entre esses países, 
onde são detectados cerca de 47.000 novos casos a cada ano, e é o segundo com o maior número de casos novos registrados no mundo, representando, assim, um grave problema de saúde pública ${ }^{5}$.

A doença pode atingir pessoas de todas as idades, de ambos os sexos, porém raramente ocorre em crianças. $\mathrm{O}$ homem é considerado a única fonte de infecção. O contágio se dá através de contato direto com a pessoa doente não tratada. A principal via de eliminação do bacilo e a mais provável porta de entrada no organismo são as vias aéreas superiores².

Algumas pessoas não adoecem quando infectadas, pois o grau variável de imunidade contra o bacilo é o que determina o adoecimento ou não do indivíduo. Nas pessoas que apresentam baixa imunidade a doença manifesta-se inicialmente através de lesões de pele, com manchas esbranquiçadas ou avermelhadas, que apresentam perda de sensibilidade tátil, térmica e dolorosa, sem evidência de lesão nervosa troncular. Estas lesões de pele ocorrem em qualquer região do corpo, mas com maior frequência na face, orelhas, nádegas, braços, pernas, costas e, em alguns casos, acometem também a mucosa nasal².

Com a evolução da doença não tratada, manifestam-se as lesões nos nervos, principalmente nos troncos periféricos ${ }^{2}$. O bacilo tem predileção pelas células de Schwann, que envolvem os axônios ${ }^{6}$. A neuropatia da hanseníase é clinicamente uma neuropatia mista, que compromete fibras nervosas sensitivas, motoras e autonômicas e podem levar à incapacidades. Os nervos envolvidos com maior frequência são o nervo facial (VII par craniano), nervo trigêmeo (V par craniano), nervo ulnar, nervo mediano, nervo radial, nervo fibular comum e nervo tibial ${ }^{5}$.

Essas manifestações podem ser acompanhadas por dor intensa, hipersensibilidade neural, edema, déficit motor e sensitivo. Algumas neurites podem se desenvolver sem dor, fenômeno chamado de neurite silenciosa ${ }^{5}$.

Os estados reacionais (ER) também são reconhecidos como causas de lesões dos nervos e de incapacidades provocadas pela hanseníase. Esses correspondem a reações do sistema imunológico do doente ao $M$. leprae. Os ER apresentam-se através de episódios inflamatórios agudos e subagudos, e são divididos em dois tipos. No tipo 1, ou reverso, o quadro clínico se caracteriza por apresentar novas lesões dermatológicas, edema nas lesões antigas e dor ou espessamento dos nervos. Já no tipo 2, o quadro clínico se manifesta principalmente como eritema nodoso hansênico (ENH), que se caracteriza por apresentar nódulos vermelhos e dolorosos, febre, dores articulares, dor e espessamento nos nervos e mal-estar generalizado².

O diagnóstico da hanseníase é realizado através do exame clínico, quando se busca os sinais dermatoneurológicos da doença. Um caso de hanseníase ao ser confirmado requer tratamento quando a pessoa apresenta uma ou mais de uma das seguintes características: lesão (ões) de pele com alteração de sensibilidade; acometimento de nervo(s) com espessamento neural; baciloscopia positiva ${ }^{2}$.

Os indivíduos são classificados de acordo com a classificação operacional de Madrid $^{5}$ em paucibacilares (PB) ou multibacilares (MB). Os PB são aqueles casos com até cinco lesões de pele, que correspondem às formas tuberculóide $\mathrm{e}$ indeterminada. Os MB são os casos com mais de cinco lesões de pele, que correspondem às formas virchowiana e dimorfa. O diagnóstico da doença e a classificação operacional são imprescindíveis para a escolha do esquema de tratamento adequado a cada caso $^{5}$.

O tratamento específico da pessoa com hanseníase, indicado pelo Ministério da Saúde, é a poliquimioterapia (PQT), que mata o bacilo, evita a evolução da doença, previne incapacidades e deformidades, levando à cura. O bacilo morto é incapaz de infectar outras pessoas, rompendo a cadeia epidemiológica da doença².

No entanto, se no momento do diagnóstico o indivíduo já apresentar alguma deformidade física 
instalada, esta pode ficar como sequela permanente no momento da alta. Essas incapacidades e deformidades causam limitação da atividade e restrição de participação social do indivíduo?

O Ministério da Saúde (MS) em $2002^{2}$ implantou a atual classificação dos graus de incapacidade, considerando como incapacidade somente as lesões em mãos, pés e olhos, por serem mais severas para as atividades cotidianas e de diagnóstico mais simples. Assim, as lesões incapacitantes dessas regiões anatômicas são graduadas conforme sua gravidade em ausente (0), moderada (1), e grave (2). O MS preconiza que os serviços de saúde devem realizar essa avaliação nos indivíduos com hanseníase no momento do diagnóstico, no mínimo uma vez ao ano durante o tratamento, e no momento da alta8.

A prevenção de incapacidades físicas começa com o diagnóstico precoce e reconhecimento da doença, tratamento correto para cada tipo de ER e das complicações, e identificação de pacientes com riscos de desenvolver complicações secundárias.

O Ambulatório Municipal de Pneumologia e Dermatologia Sanitária (AMPDS) do município de Guarapuava-PR tem atuado nas ações de controle da hanseníase conforme preconizado pelo MS, contudo, ainda há casos de grau 2 de incapacidade física (GIF2) no momento do diagnóstico. Sendo assim, um estudo epidemiológico torna-se de extrema importância para colaborar no controle da doença e na prevenção de incapacidades.

Diante disso, o presente estudo objetivou traçar o perfil dos casos novos de hanseníase que apresentavam GIF2 no momento do diagnóstico, descrever os tipos de incapacidades mais comuns e as regiões anatômicas mais suscetíveis a essas incapacidades.

\section{METODOLOGIA}

Foi realizado um estudo transversal epidemiológico, com início após a autorização da Secretaria Municipal de Saúde do município de Guarapuava - PR e aprovação do Comitê de Ética em Pesquisa do Setor de Ciências da Saúde (COMEP) da Universidade Estadual do CentroOeste (UNICENTRO,) sob o parecer de número 552.011. Foi obedecida a resolução 466/2012 do Conselho Nacional de Saúde.

Os instrumentos de pesquisa utilizados foram os prontuários dos pacientes, dados do Sistema de Informação de Agravos de Notificação - SINAN/ DATASUS, registros em livros ata nos arquivos do Ambulatório Municipal de Pneumologia e Dermatologia Sanitária (AMPDS), centro de referência no atendimento das ações de controle da hanseníase em Guarapuava-PR. Foram selecionados para a pesquisa os casos novos de hanseníase que apresentavam GIF 2 no momento do diagnóstico, no período de janeiro de 2003 a dezembro de 2012. Em nenhum momento da pesquisa os indivíduos foram identificados, sendo assim, não houve a necessidade do uso do Termo de Consentimento Livre e Esclarecido.

Os dados foram coletados buscando as variáveis: sexo, raça, idade, escolaridade, data do diagnóstico, forma clínica da doença, modo de entrada no atendimento, modo de detecção do caso novo, grau de incapacidade física no momento do diagnóstico e no final do tratamento, de acordo com o formulário de classificação de incapacidade física ${ }^{5}$.

Para fins de análises no presente estudo, a população de Guarapuava-PR foi verificada no site do Instituto Brasileiro de Geografia e Estatística IBGE ${ }^{10}$, nos anos de estudo, conforme segue: 2003 - 160.932 habitantes, 2004 - 164.772 habitantes, 2005 - 166.897 habitantes, 2006 - 169.007 habitantes, 2007 - 164.567 habitantes, 2008 171.230 habitantes, 2009 - 172.728 habitantes, 2010 - 167.328 habitantes, 2011 - 168.349 habitantes e 2012 - 169.252 habitantes.

Os dados foram inseridos em planilha Microsoft Office Excel 2007, e as análises estatísticas foram realizadas utilizando o software estatístico SPSS 21.0. Os dados foram apresentados em média 
e desvio padrão. Inicialmente foi empregado o teste de Shapiro-Wilk para verificar a distribuição das variáveis. Devido ao não atendimento do pressuposto de normalidade recorreu-se à estatística não paramétrica, empregando-se o teste U de Mann-Whitney nas comparações com dois fatores. Foi utilizado o teste de homogeneidade das variâncias para comparações com mais de três fatores, e também foi utilizado ANOVA oneway nos casos de homogeneidade das variâncias e Kruskal-Wallis para distribuição não paramétrica. Para os dados nominais utilizou-se o teste Quiquadrado. Para todos os testes a significância adotada foi de $p<0,05$.

\section{RESULTADOS}

Durante janeiro de 2003 a dezembro de 2012. período de 10 anos considerado para este estudo, foram diagnosticados 307 indivíduos com hanseníase no município de Guarapuava - PR. Destes, 45 (14,65\%) foram classificados com o grau dois de incapacidade (GIF2) no início do tratamento, de acordo com o formulário de classificação de incapacidade física ${ }^{5}$. 0 ano de 2004 apresentou o maior coeficiente de detecção de novos casos de hanseníase (26,70/100.000 habitantes), e o maior número de indivíduos diagnosticados com GIF2 (24,4\%). Esses dados podem ser visualizados na tabela 1.

O sexo masculino foi acometido em $75,6 \%$ dos casos analisados, e o femininos em $24,4 \%$. A idade média mais prevalente foi 61,09 anos para homens e 53,97 anos para mulheres. Não houve significância estatística entre a idade média do gênero feminino e masculino $(p=0,19)$.

Na tabela 2 é possível verificar a cor de pele, a escolaridade, a zona de residência, forma clínica da doença e a classificação operacional. 0 estudo constatou que os indivíduos da área rural são significativamente mais jovens que os da área urbana $(p=0,046)$. Não houve diferença estatística entre a idade média dos casos MB e PB $(p=0,7)$. No que diz respeito à baciloscopia no momento do diagnóstico, em 29 casos (64,4\%) foram positivas.

Tabela 1. Distribuição anual dos casos novos de hanseníase e com GIF2, de 2003 a 2012, em Guarapuava-PR

\begin{tabular}{cccccc}
\hline $\begin{array}{c}\text { Ano do } \\
\text { diagnóstico }\end{array}$ & $\begin{array}{c}\text { População de } \\
\text { Guarapuava }\end{array}$ & $\begin{array}{c}\text { Número } \\
\text { total de } \\
\text { casos novos }\end{array}$ & $\begin{array}{c}\text { Coeficiente de } \\
\text { detecção da } \\
\text { população geral }\end{array}$ & $\begin{array}{c}\text { Número de } \\
\text { casos novos } \\
\text { com GIF2** }\end{array}$ & $\begin{array}{c}\% \text { GIF2 } \\
\text { início do } \\
\text { tratamento }\end{array}$ \\
$\mathbf{2 0 0 3}$ & 160.932 & 42 & 26,09 & 8 & 19,04 \\
$\mathbf{2 0 0 4}$ & 164.772 & 44 & 26,70 & 11 & 25 \\
$\mathbf{2 0 0 5}$ & 166.897 & 37 & 22,16 & 6 & 16,21 \\
$\mathbf{2 0 0 6}$ & 169.007 & 27 & 15,97 & 2 & 7,40 \\
$\mathbf{2 0 0 7}$ & 164.567 & 35 & 21,26 & 4 & 11,42 \\
$\mathbf{2 0 0 8}$ & 171.230 & 30 & 17,52 & 2 & 6,66 \\
$\mathbf{2 0 0 9}$ & 172.728 & 30 & 17,36 & 5 & 16,66 \\
$\mathbf{2 0 1 0}$ & 167.328 & 18 & 10,75 & 1 & 5,55 \\
$\mathbf{2 0 1 1}$ & 168.349 & 19 & 11,28 & 2 & 10,52 \\
$\mathbf{2 0 1 2}$ & 169.252 & 25 & 8,86 & 4 & 16 \\
\hline Total & - & 307 & - & 45 & 14,65 \\
\hline
\end{tabular}

*Coeficiente de detecção: Casos novos/população total de Guarapuava-PR x 100.000;

${ }^{*}$ GIF2:Grau de incapacidade física dois 
Tabela 2. Características dos indivíduos, separados pelo gênero, dos casos novos de hanseníase, de 2003 a 2012, em Guarapuava-PR

\begin{tabular}{|c|c|c|c|c|}
\hline \multicolumn{2}{|c|}{ Caracteristicas } & \multirow{2}{*}{$\begin{array}{c}\mathbf{F}^{*} \\
8\end{array}$} & \multirow{2}{*}{$\begin{array}{l}\mathbf{M}^{\star *} \\
28\end{array}$} & \multirow[t]{2}{*}{ p-valor } \\
\hline \multirow{3}{*}{ Raça/cor } & Branca & & & \\
\hline & Parda & 2 & 4 & \multirow[t]{2}{*}{0,78} \\
\hline & Negra & 1 & 2 & \\
\hline \multirow{8}{*}{ Escolaridade } & Analfabeto & 4 & 8 & \multirow{8}{*}{0,80} \\
\hline & 1-4 anos & 5 & 16 & \\
\hline & 4 anos & 1 & 2 & \\
\hline & 4-8 anos & 1 & 6 & \\
\hline & 8 anos & 0 & 1 & \\
\hline & $>8$ anos & 0 & 1 & \\
\hline & Ensino médio Completo & 0 & 0 & \\
\hline & Graduação & 0 & 0 & \\
\hline \multirow[b]{2}{*}{ Zona de residência } & Urbana & 10 & 28 & \multirow[b]{2}{*}{0,83} \\
\hline & Rural & 1 & 6 & \\
\hline \multirow{2}{*}{ Classificação Operacional } & Multibacilar & 9 & 31 & \multirow{2}{*}{0,75} \\
\hline & Paucibacilar & 2 & 3 & \\
\hline \multirow{3}{*}{ Forma Clínica } & Tuberculoide & 4 & 1 & \multirow{3}{*}{0,03} \\
\hline & Dimorfa & 5 & 14 & \\
\hline & Virchowiana & 2 & 19 & \\
\hline
\end{tabular}

${ }^{*} F$ : feminino; ${ }^{* *} M$ : masculino

Em relação ao modo de detecção, 19 (42,2\%) casos eram encaminhamento, 11 (24,5\%) demanda espontânea, 1 (2,2\%) por exame de coletividade, $3(6,6 \%)$ por exame de contato e 11 (24,5\%) por outros motivos.

No momento do diagnóstico, 44 (97,7\%) dos casos apresentavam alterações de sensibilidade associadas ao GIF2. Os locais mais afetados pelas incapacidades físicas causadas pela doença no início e após o tratamento medicamentoso podem ser visualizados na tabela 3.

As lesões tróficas ou traumáticas e as garras foram as principais incapacidades encontradas, tanto no momento do diagnóstico, quanto após o tratamento medicamentoso. Os pés foram mais acometidos pelas lesões tróficas ou traumáticas, e as mãos mais acometidas pelas garras, conforme a tabela 4.

Após o término do tratamento medicamentoso, não foi possível realizar a reavaliação em 1 indivíduo antes da conclusão do tratamento, por motivo de falecimento. Dos 44 indivíduos reavaliados, 5 $(11,4 \%)$ evoluíram para grau 0 de incapacidade, 12 (27,2\%) evoluíram para o grau 1 de incapacidade, e 27 (61,4\%) permaneceram com o grau dois de incapacidade.

\section{DISCUSSÃO}

A hanseníase é uma patologia com alto poder incapacitante que pode acarretar graves 
Tabela 3. Regiões anatômicas afetadas pelas incapacidades no início e fim do tratamento

\begin{tabular}{ccccccc}
\hline & \multicolumn{3}{c}{ Inicio do tratamento } & \multicolumn{3}{c}{ Conclusão do tratamento } \\
\hline Segmento anatômico & $\mathrm{F}^{*}$ & $\mathrm{M}^{* *}$ & $\mathrm{p}$-valor & $\mathrm{F}^{*}$ & $\mathrm{M}^{* *}$ & $\mathrm{p}$-valor \\
Pés & 00 & 09 & & 00 & 09 & \\
Mãos & 01 & 03 & 01 & 02 & \\
Olhos & 00 & 00 & & 00 & 02 & 14 \\
Pés e mãos & 06 & 16 & 0,71 & 06 & 0,38 \\
Pés e olhos & 03 & 03 & & 02 & 01 & \\
Mãos e olhos & 00 & 00 & & 00 & 01 & \\
Mãos, pés e olhos & 01 & 03 & 01 & 00 & \\
\hline
\end{tabular}

${ }^{\star}$ F: feminino; ${ }^{* \star M}$ : masculino

Tabela 4. Relação entre o tipo de incapacidade e a região anatômica afetada

\begin{tabular}{|c|c|c|c|c|c|c|c|c|}
\hline & \multicolumn{2}{|c|}{ Mãos } & \multicolumn{2}{|c|}{ Pés } & \multicolumn{2}{|c|}{ Mãos e Pés } & \multicolumn{2}{|c|}{ Olhos } \\
\hline & $I^{*}$ & $F^{* *}$ & $I^{*}$ & $F^{* *}$ & $1^{*}$ & $F^{* *}$ & $1^{*}$ & $F^{* *}$ \\
\hline Lesões Tróficas e/ou traumáticas & 8 & 5 & 15 & 6 & 7 & 4 & - & - \\
\hline Garras & 15 & 12 & 5 & 4 & 4 & 3 & - & - \\
\hline Reabsorção & 1 & 0 & 4 & 4 & 0 & 0 & - & - \\
\hline Pé/Mão Caido & 1 & 0 & 3 & 1 & 0 & 0 & - & - \\
\hline Logoftálmico e/ou ectrópio & - & - & - & - & - & - & 4 & 3 \\
\hline Triquiase & - & - & - & - & - & - & 1 & 1 \\
\hline Opacidade & - & - & - & - & - & - & 3 & 3 \\
\hline Acuidade visual Menor $\mathbf{1 , 0}$ & - & - & - & - & - & - & 2 & 2 \\
\hline
\end{tabular}

*I: inicio do tratamento medicamentoso; ${ }^{* *} \mathrm{~F}$ : fim do tratamento medicamentoso

problemas ao portador, como a diminuição da capacidade de trabalho, a limitação da vida social e problemas psicológicos ${ }^{11,12}$. A classificação do grau de incapacidade pode ser utilizada como um indicador da precocidade do diagnóstico. ${ }^{8}$ Além disso, pode determinar a qualidade do atendimento ao indivíduo portador de hanseníase através da avaliação inicial e final do tratamento, e ainda contribuir para um melhor planejamento das ações de combate à doença.

No estado do Paraná, nos anos de 2001 a 2008, a GIF2 oscilou entre 8\% e 14,3\%, apresentando classificação de média para alta no período, segundo parâmetros inseridos na Programação de Ações Prioritárias de Vigilância em Saúde - PAVS ${ }^{13}$. Números próximos foram encontrados em estudo no extremo sul de Santa Catarina ${ }^{14}$ e na presente pesquisa, em que a GIF2 correspondeu a 14,65\% (oscilando de 5\% a 19\%) nos novos casos no momento do diagnóstico em todos os anos analisados.

O ano de 2004 foi o que apresentou um maior 
número de diagnosticados, assim como no estudo realizado por Moreira e colaboradores ${ }^{15}$. Nesse ano, ocorreu uma campanha nacional lançada pelo Ministério da Saúde chamada "Vamos remover essa mancha Brasil", que mobilizou vários estados com informações sobre a doença e como identificála. Também nesse ano as ações de controle da hanseníase foram descentralizadas para a atenção básica, fatos que podem explicar o grande número de diagnósticos ${ }^{15}$.

A prevalência de incapacidades causadas pela hanseníase está correlacionada às condições socioeconômicas, culturais, de escolaridade, e de acesso a informações e serviços de saúde. Estudos demonstram que a doença vem ocorrendo com maior frequência em classes menos favorecidas, com menor escolaridade $3,15,16,17$.

O baixo nível de escolaridade pode diminuir a compreensão das diretrizes de prevenção da doença, bem como a compreensão sobre o tratamento adequado e cuidados médicos necessários ${ }^{18}$. Existe relação estatisticamente significativa entre indivíduos com baixa escolaridade e limitação das atividades causadas pela doença ${ }^{3.16}$, fato que condiz com os achados nessa pesquisa.

As incapacidades e limitações nas atividades têm se demonstrado com maior frequência nos casos multibacilares $(\mathrm{MB})^{3.19,20,21 .}$, corroborando com os dados da presente pesquisa, em que houve prevalência de casos $\mathrm{MB}$ correspondendo a $88,8 \%$ dos casos com GIF2. Dentre esses, a forma virchowiana apareceu com maior frequência, seguida pela forma dimorfa, convergindo com outros estudos já realizados 22.19 .

Essas formas clínicas são reconhecidas pelo grande poder de transmissibilidade e elevado indice de incapacidade residual ${ }^{21}$. Moschioni e

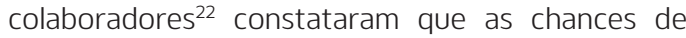
desenvolvimento de GIF2 em indivíduos com forma clínica virchowiana e dimorfa é 16,5 e 12,8 vezes maior, respectivamente, quando comparados à forma indeterminada, que corresponde à forma inicial da patologia. Além disso, a forma MB é um importante indicador do diagnóstico $\operatorname{tardio}^{23}$, e possui maior incidência no sexo masculino 18,21,24,25.

A doença não tem predileção por sexo ${ }^{5}$ porém, no presente estudo houve prevalência do sexo masculino, corroborando com estudos anteriores $16,17,20,25-33$ e com dados da Organização Mundial da Saúde, que afirmam que na maior parte do mundo os homens são mais afetados que as mulheres, em uma proporção de 2:13.

Em contrapartida, alguns autores apontam a prevalência do sexo feminino 14,21,24.34, e o número de mulheres diagnosticadas tende a aumentar devido a mudanças culturais e sociais que geram maior exposição do gênero ${ }^{15}$. As mulheres também têm maior preocupação com a estética corporal, assim procuram os serviços de saúde com maior frequência, o que contribuiu com o diagnóstico precoce da doença18.

O sexo masculino, por outro lado, além de se preocupar menos com estética corporal, mantém maior contato social, frequente exposição a ambientes de risco, dificuldade para chegar à unidade de saúde durante seu dia de trabalho, medo de perder o emprego por conta do estigma causado pela doença, o que leva ao diagnóstico tardio e presença de incapacidades no momento do diagnóstico ${ }^{11,14,28,35}$.

Quanto à cor de pele, a prevalência nesse estudo foi da cor branca. As características regionais devem ser consideradas ao analisar esta característica, pois em Guarapuava-PR há predominância de cor de pele mais acometida branca $^{36}$. Em estudo realizado no Nordeste do Brasil $^{18}$, a predominância foi de pessoas pardas, cor de predominância na população geral daquela região.

A hanseníase é considerada uma doença de adultos pelo longo período de incubação, mas crianças também são suscetíveis ${ }^{37}$. Casos em menores de 15 anos indicam uma maior gravidade da endemia24,32,37,38. Não houve casos nesta faixa etária nesse trabalho. Várias pesquisas apontam que a doença predomina na faixa etária economicamente ativa, ocorrendo grande 
impacto socioeconômico 21,24,25,31,37. Alguns trabalhos detectaram presença maior de deformidades conforme o aumento da idade ${ }^{8.21}$, reforçando os achados da presente pesquisa, onde a faixa etária mais acometida foi a de 61 a 70 anos.

Nesse estudo os indivíduos da zona rural são significativamente mais jovens que os da área urbana. Porém os indivíduos da área urbana são mais afetados pelo GIF2. A prevalência em áreas urbanas ocorre devido à maior densidade populacional e aumento da taxa de pobreza existente nessas áreas ${ }^{17}$.

Vários dos indivíduos analisados neste estudo apresentaram mais de uma região anatômica afetada pelas incapacidades físicas no momento do diagnóstico. Indivíduos que tinham alterações em mãos e pés apareceram com maior frequência, sendo os pés as regiões mais afetadas, seguidos pelas mãos. As alterações nos olhos foram as menos frequentes, conforme outros achados na literatura ${ }^{11,19,39}$.

Quanto ao tipo de acometimento, no momento do diagnóstico, as lesões tróficas ou traumáticas foram as que apareceram com maior frequência, seguido pelas garras. As úlceras cutâneas constituem uma importante complicação consequente da neuropatia nos portadores de hanseníase, e o local comumente afetado é a região plantar $^{40}$.

A região plantar é comumente afetada devido às alterações biomecânicas e diminuição da sensibilidade. A alteração biomecânica ocorre a partir de amiotrofias, fraquezas musculares e deformidades, que contribuem diretamente para o desarranjo ósseo do pé, o que faz com que o indivíduo realize uma marcha desajustada, e provoque novos pontos de pressão em regiões do pé não apropriadas. Além disso, o portador de hanseníase possui diminuição ou abolição da sensibilidade, o que reflete na diminuição da proteção fisiológica necessária para a prevenção de inúmeras lesões cutâneas ${ }^{40}$.

As lesões cutâneas devem ser abordadas e devidamente prevenidas, pois constituem porta de entrada para infecções, que podem se agravar e conduzir o indivíduo a complicações graves, até mesmo à amputação ${ }^{41}$. Da mesma forma acontece com as garras, que se não abordadas precocemente, podem resultar em instalação permanente de deformidades físicas, fazendo com que o indivíduo lesado ou traumatizado perca sua capacidade funcional. As garras em mãos dificultam a preensão de objetos e a função das mãos, acarretando em um sério problema tanto de produtividade e qualidade de vida, quanto socioeconômico ao indivíduo afetado ${ }^{42}$.

Neste estudo houve uma redução nos casos de GIF2 após o término do tratamento medicamentoso. Apesar disso, 61,4\% dos indivíduos ainda apresentavam GIF2 no momento da cura da doença, números parecidos com o do estudo realizado no estado do Paraná, durante os anos de 2001 e $2008^{13}$. em que a GIF2 estava presente em $63,2 \%$ dos casos. Esses resultados são considerados precários, segundo parâmetros inseridos na Programação de Ações Prioritárias de Vigilância em Saúde - PAVS, e devem ser considerados pelos profissionais de saúde.

O percentual de incapacidades, nos casos novos, é um indicador de extrema importância para avaliação do combate da hanseníase, pois pode determinar a oportunidade da detecção ou a fase de controle da endemia em uma localidade ${ }^{43,44}$. Quando este indicador está aumentado, aponta para prevalência oculta e diminuição do diagnóstico precoce ${ }^{43,23}$.

Durante o tratamento PQT e após a alta, o profissional de saúde deve ter uma atitude de vigilância em relação ao potencial incapacitante da doença, visando diagnosticar precocemente e tratar adequadamente as neurites e reações, a fim de prevenir incapacidades e evitar que as já existentes evoluam para deformidades².

Diante do exposto, a fisioterapia desempenha um papel importante na equipe multidisciplinar no acompanhamento do portador de hanseníase. O AMPDS de Guarapuava-PR conta com esse profissional. O fisioterapeuta deve participar 
ativamente da avaliação, prevenção, tratamento e reabilitação dessas incapacidades físicas, durante e após o tratamento medicamentoso, com o objetivo de prevenir a expansão da doença, realizar diagnóstico precoce, evitar as incapacidades e reintegrar os indivíduos já afetados na sociedade ${ }^{45,46}$

\section{CONCLUSÃO}

O estudo constatou que homens com mais de 60 anos que residem em zona urbana e homens de idade produtiva em zona rural, com baixa escolaridade e cor de pele branca, apresentaram diagnóstico tardio com lesões incapacitantes. Os pés foram as regiões anatômicas mais acometidas por lesões tróficas ou traumáticas e as mãos foram as regiões mais acometidas pelas garras. Há necessidade de intensificar a busca ativa de afetados pela doença em locais frequentados por indivíduos com esse perfil.

Após o tratamento medicamentoso houve uma redução do grau de incapacidade em 38,3\% dos casos, e ocorreu uma diminuição na série histórica da detecção dos casos de grau dois de incapacidade no momento do diagnóstico, demonstrando relativa efetividade da atuação do AMPDS do município de Guarapuava-PR junto às unidades descentralizadas, e o trabalho de intersetorialidade, quanto às ações de controle da hanseníase.

\section{REFERÊNCIAS}

1. Batista ES, Campos RX, Queiroz RCG, Siqueira SL, Pereira SM, Pacheco TJ, et al. Perfil sociodemográfico e clínico-epidemiológico dos pacientes diagnosticados com hanseníase em Campos dos Goytacazes, RJ. Rev Bras Clín Méd. 2011; 9(2): 101-6.

2. Ministério da Saúde, Departamento da Atenção Básica. Guia para o controle da hanseníase. Brasília (DF); 2002.

3. World Health Organization. Weekly epidemiological record [Internet]. Geneva: World Health Organization; 2017 [cited 2018 Jan 17]. 22 p. Available from: http://apps.who.int/iris/ bitstream/10665/255149/1/WER9217.pdf

4. Ikehara E, Nardi SMT, Ferrigno ISV, Pedro HSP, Paschoal VD. Escala Salsa e grau de Incapacidades da Organização Mundial de Saúde: avaliação da limitação de atividades e deficiência na hanseníase. Acta fisiatra. 2010; 17(4): 169-74.

5. Ministério da Saúde, DATASUS. Informação em Saúde Epidemiológica e morbidade. Hanseníase [Internet]. 2017 [citado 2018 jan 17]. Disponivel em www2.datasus.gov.br/DATASUS/index. php?area $=0203 \&$ id $=31032752$

6. Pucci FH. Teófilo, CR, Aragão SGA, Távora LGF. A dor no paciente com hanseníase. Rev Dor. 2011; 12(1): 15-8.

7. Raposo MT, Raposo AVC, González MAS, Medeiros JLA, Nemes MIB. Avaliação de incapacidades em pessoas vivendo com hanseníase: análise do grau de incapacidade em Campina Grande Paraíba. Cad. Saúde Coletiva. 2009:17(1): 221-33.

8. Alves CJM, Barreto J A, Fogagnolo L, Contin LA, Nassif PW Avaliação do grau de incapacidade dos pacientes com diagnóstico de hanseníase em Serviço de Dermatologia do Estado de São Paulo. Rev Soc Bras Med Trop. 2010:43(4):460-61.

9. Sales AM, Campos DP, Hacker MA, Nery JAC, Deuppre NC. Rangel E, Euzenir, et al. Progression of leprosy disability after discharge: is multidrug therapy enough? Rev Trop Med and Int Health. 2011; 8(9): 145-53.

10. Instituto Brasileiro de Geografia e Estatística - IBGE Estimativa de população [Internet]. 2012 [citado 2018 Out 22]. Disponível em http://www.ibge.gov.br/home/estatistica/ populacao/estimativa2012/default.shtm

11. Brakel WHV, Sihombing B, Djarir H, Beise K, Kusumawardhan L, Yulihane R, et al. Disability in people affected by leprosy: the role of impairment, activity, social participation, stigma and discrimination. Citation: Glob Health Action. 2012;5:183-94.

12. Rao PSS, Darlong F, Timothy M, Kumar S, Abraham S,Kurian R. Adjusted life years for incapacity for work (DAWLYS) leprosy affected people in India. Indiano J Med Res. 2013:137(5):907-10.

13. Ministério da Saúde, Secretaria de Vigilância em Saúde Departamento de Vigilância Epidemiológica. Hanseníase no Brasil, dados e indicadores selecionados. Brasilia (DF): Ministério da Saúde, 2009.

14. Melão S, Blanco LFO, Mounzer N, Veronezi CCD, Simões PWTA Perfil epidemiológico dos pacientes com hanseníase no extremo sul de Santa Catarina, no período de 2001 a 2007. Rev Soc Bras Med Trop. 2011:44(1):79-84

15. Moreira SC, Batos CJC, Tawil L. Epidemiological situation of leprosy in Salvador from 2001 to 2009. An Bras Dermatol. 2014:89(1):107-17

16. Hacker MA, Sales AM, Illarramendi X, Nery JA, Duppre NC Bastos FI, et al. A profile of patients treated at a national leprosy outpatient referral clinic in Rio de Janeiro, Brazil, 1986-2007. Rev Panam Salud Publica. 2012;31(6):485-91.

17. Entezarmahdi R, Majdzadeh R, Foroushani AR, Nasehi $M$ Lameei A,Naieni KH. Inequality of Leprosy Disability in Iran, Clinical or Socio-Economic Inequality: an extended concentration index decomposition approach. Int J Prev Med. 2014;5(4):414-23.

18. Corrêa RGCF, Aquino DMC, Caldas AJM, Amaral DKCR, França FS, Mesquita ERRBP. Epidemiological, clinical, and operational aspects of leprosy patients assisted at a referral service in the state of Maranhão, Brazil. Rev Soc Bras de Med Trop. 2012:45(1):89-94

19. Sarkar J, Dasgupta A, Dutt D. Incapacidade funcional entre casos novos de hanseníase, uma questão de preocupação: um estudo baseado instituição em um distrito endêmico de hanseníase no estado de Bengala Ocidental, na Índia. Indiano J Dermatol Venereol Leprol. 2012;78: 328-34. 
20. Antonio JR, Soubhia RMC, Paschoal VD, Oliveira GB de, Rossi NCP. Maciel MG, et al. Avaliação epidemiológica dos estados reacionais e incapacidades físicas em pacientes com hanseníase na cidade de São José do Rio Preto. Arq Ciênc Saúde 2011:18(1):9-14.

21. Ribeiro Júnior AF, Vieira MA, Caldeira AP. Perfil epidemiológico da hanseníase em uma cidade endêmica no Norte de Minas Gerais. Rev Bras Clin Med.2012:10(4):272-77.

22. Moschioni C, Antunes CMFA, Grossi MAF, Lambertucci JR. Risk factors for physical disability at diagnosis of 19,283 new cases of leprosy. Rev Soc Bras de Med Trop.2010;43(1):19-22.

23. Hacker MA, Duppre NC, Nery JAC, Sales AM, Sarno EN. Characteristics of leprosy diagnosed through the surveillance of contacts: a comparison with index cases in Rio de Janeiro, 19872010. Mem Inst Oswaldo Cruz. 2012;107(1): 49-54.

24. Souza VB, Silva MRF, Silva LMS, Torres RAM, Gomes KWL, Fernandes MC et al. Perfil epidemiológico dos casos de hanseníase de um centro de saúde da família. Rev Bras Promoção Saúde. 2013:26(1):110-16

25. Miranzi SSC, Pereira LHM, Nunes AA. Perfil epidemiológico da hanseníase em um município brasileiro, no período de 2000 a 2006. Rev Soc Bras Med Trop. 2010:43(1): 62-7.

26. Monteiro LD, Alencar CH, Barbosa JC, Novaes CCBS, Silva RCP. Heukelbach J. Pós-alta de hanseníase: limitação de atividade e participação social em área hiperendêmica do Norte do Brasil. Rev Bras Epidemiol. 2014:91-104.

27. Lanza FM. Cortez DN, Gontijo LT, Rodrigues JSJ. Perfil epidemiológico da hanseníase no município de Divinópolis, Minas Gerais. Rev Enferm UFSM. 2012:2(2):365-74.

28. Monteiro LD, Alencar CHM, Barbosa JC, Braga KP, Castro MD de, Heukelbach J. Incapacidades físicas em pessoas acometidas pela hanseníase no período pós-alta da poliquimioterapia em um município no Norte do Brasil. Cad Saúde Pública. 2013:29(5):90920.

29. Imbiriba EB, Basta PC, Pereira ES, Levino A, Garnelo L. Hanseníase em populações indígenas do Amazonas, Brasil: um estudo epidemiológico nos municípios de Autazes, Eirunepé e São Gabriel da Cachoeira (2000 a 2005). Cad Saúde Pública. 2009:25(5):972-84

30. Castro RNC de, Costa Veloso T da, Matos Filho LJS, Coelho LC. Pinto LB, Castro AMNC de. Avaliação do grau de incapacidade física de pacientes com hanseníase submetidos ao Dermatology Quality Life Index em Centro de Referência e Unidades Básicas de Saúde de São Luis, MA. Rev Bras Clin Med. 2009;7:390-2.

31. Ramos JM, Martin MM, Reis F, Lemma D, Belinchón I, Gutiérrez F. Gender differential on characteristic and outcome of leprosy patients admitted to a long-term care rural hospital in SouthEastern Ethiopia. Int J Equity Health. 2012;11(56):2-7.

32. Sousa MWG de, Silva DC, Carneiro LR, Almino MLBF, Costa ALF da. Epidemiological Profile of Leprosy in the Brazilian state of Piaui between 2003 and 2008*. An Bras Dermatol. 2012; 87(3): 389-5.

33. Pinto RA, Maia HF, Silva MAF, Marback M. Perfil clínico e epidemiológico dos pacientes notificados com hanseníase em um hospital especializado em Salvador, Bahia. Rev Baiana Saúde Publica. 2010;34(4):906-18.

34. Nardil SMT, Paschoal VD, Chiaravalloti Neto F, Zanetta DMT. Deficiências após a alta medicamentosa da hanseníase: prevalência e distribuição espacial. Rev Saúde Pública. 2012:46(6):969-77.
35. Ferreira SMB, Ignotti E, Gamba MA. Características clínicolaboratoriais no retratamento por recidiva em hanseníase. Rev Bras Epidemiol. 2012:15(3):573-8.

36. Instituto Brasileiro de Geografia e Estatística - IBGE. Diretoria de Pesquisas Coordenação de População e Indicadores Sociais: sintese de indicadores sociais, uma análise de condições de vida da população brasileira. Rio de Janeiro: IBGE; 2010.

37. Lima HMN, Sauaia N, Costa VRL da, Coelho Neto GT, Figueiredo PMS. Perfil epidemiológico dos pacientes com hanseníase atendidos em Centro de Saúde em São Luís, MA. Rev Bras Clin Med. 2010:8(4):323-7.

38. Mastrangelo G, Scoizzato L, Fadda E, Silva GV da, Santos LJ, Cegolon L. Epidemiological pattern of leprosy in an endemic area of North-East Brazil, 1996-2005: the supporting role of a Nongovernmental Organization. Rev Soc Bras de Med Trop. 2009;42(6):629-32.

39. Guerrero MI, Muvdi S, León Cl. Retraso em el diagnóstico de lepra como fator pronóstico de discapaci- daden una cohorte de pacientes em Colombia, 2000-2010. Rev Panam Salud Publica.2013:33(2):137-43.

40. Bhatt YC, Panse NS, Vyas KA, Patel GA, Transferência de tecido livre para úlcera trófica lepra complicando. Indiano J Plast Surg. 2009:42(1):115-7.

41. Gomes FG. Frade MAC. Foss NT. Úlceras cutâneas na hanseníase: perfil clínico-epidemiológico dos pacientes. An Bras Dermatol. 2007;82(5):433-7.

42. Souza CFD, Slaibi EB, Pereira RN, Francisco FP, Bastos MLS de, Lopes MRA, et al. A importância do diagnóstico precoce da hanseníase na prevenção de incapacidade. Hansen Int. 2010;35(2):61-6

43. Conte ECM, Magalhais LCB de, Cury MRCO, Soubhia RMC, Nardi SMT, Paschoal VD, Lombardi C. Situação Epidemiológica da hanseníase no município de São José do Rio Preto, SP. Brasil. Arq Ciênc Saúde. 2009;16(4):149-54.

44. Mastrangelo G. Scoizzato L, Fadda E. Silva GV da, Jesus Santos L de, Cegolon L. Epidemiological pattern of leprosy in an endemic area of North-East Brazil, 1996-2005: the supporting role of a Nongovernmental Organization. Rev Soc Bras Med Trop. 2009:42(6):629-32.

45. Tavares JP, Barros JS, Silva KCC, Barbosa E, Reis GR, Silveira JM Fisioterapia no atendimento de pacientes com hanseníase: um estudo de revisão. Rev Amazônia. 2013:1(2):37-43.

46. Jensen RGD. Hanseníase: abordagem fisioterapêutica. Rev. Olhar. 2010:1(2):332-9. 